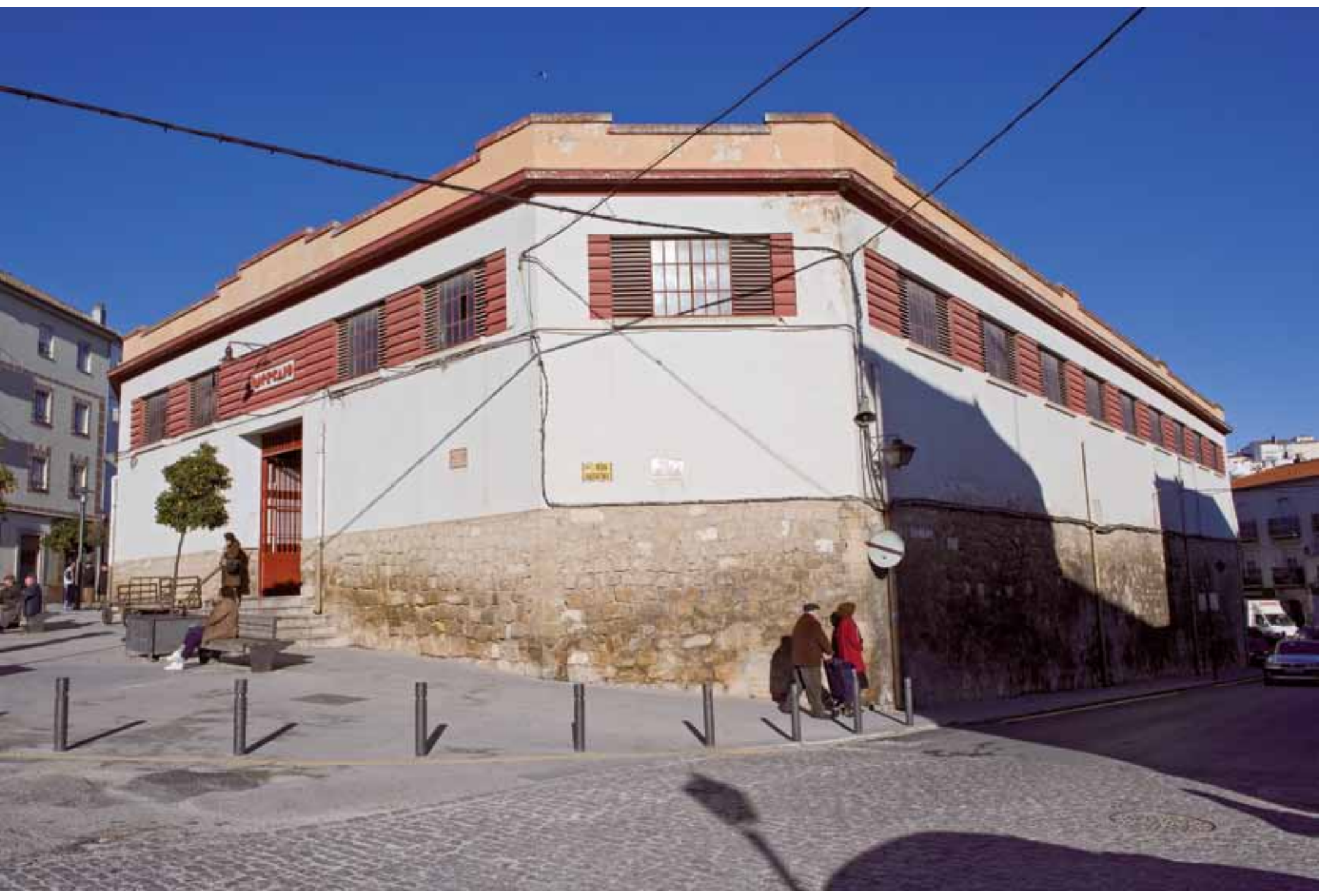

Vista exterior del mercado de abastos de Úbeda. Fuente: Fondo Gráfico IAPH 


\section{Abastos modernos. Mercados del siglo XX: la evolución de un tipo arquitectónico contemporáneo en la provincia de Jaén}

Plácido González Martínez, Centro de Documentación y Estudios del IAPH

La evolución del tipo arquitectónico del mercado es resultado de una progresiva adaptación a un espacio normativo estricto, que buscaba mejorar las condiciones de abastecimiento de la población. Al mismo tiempo, el mercado se consolidó como espacio público por excelencia en pueblos y ciudades, generador de una poderosa identidad contemporánea. Un recorrido por los mercados del siglo XX en Jaén ofrece una variada casuistica, desde las primeras experiencias vinculadas a la aparición de la cultura industrial en la provincia, hasta muestras recientes de su vocación netamente urbana.

El avance del pensamiento positivista en la ciudad industrial desde el siglo XIX tuvo como resultado la elaboración de un corpus teórico, el urbanismo, que permitiese imaginar un horizonte cualitativo para el desarrollo urbano, frente al simple proceso acumulativo y caótico que frecuentemente fue el crecimiento de las ciudades. Ideas de eficiencia, fluidez e higiene prevalecieron a la hora de definir la creación de un tejido urbano moderno, en el que se hacia evidente la necesidad de establecer un reparto equitativo de equipamientos públicos, que subvirtiesen las relaciones tradicionales de sumisión entre centro y periferia, entre el medio urbano y el territorio rural.

La nueva organización burocrática estatal y local a la hora de regular las actividades comerciales se tradujo en el control sistemático del abastecimiento de productos perecederos, en la regulación de la venta callejera tradicional y en la especialización funcional progresiva de ciertas áreas urbanas. La consolidación del mercado como tipología arquitectónica acompañó a la consolidación de una cultura del consumo de dinámicas cambiantes, en la que se imponían requisitos estrictos relativos a la higiene en los alimentos, el trasiego de mercancias y las circulaciones de público. El mercado aparece, por tanto, como testimonio histórico de estos cambios, ofreciendo, ex novo, respuestas arquitectónicas ideales que iban más allá de nuevos requisitos técnicos, y que habrian de erigirse además como alternativas a los mitos históricos del zoco, el foro y el ágora. Al definirse el mercado se definía también un espacio público de sociabilidad, portador de un importante significado identitario.
Un recorrido por los mercados andaluces muestra la condición de punta de lanza que disfrutaron para la introducción de la modernidad arquitectónica en nuestro entorno, con una capacidad de impregnación sobre el tejido urbano y los hábitos sociales que resulta hoy dia más que evidente. No es casualidad, en este sentido, que el mercado de la Puerta de la Carne en Sevilla (Gabriel Lupiáñez y Aurelio Gómez Millán, 1927-1929) haya sido generalmente señalado como primer edificio netamente racionalista de Andalucia; como tampoco es menor la trascendencia que tuvo a nivel nacional e internacional la construcción del mercado de Algeciras (Manuel Sánchez Arcas y Eduardo Torroja, 1933), aunando innovación tipológica y riesgo estructural en la forma de su liviana y osada cubierta de hormigón armado.

A la hora de documentar el patrimonio contemporáneo andaluz, la tipología de los mercados ofrece 14 ejemplos destacados entre los años 1900 y 2000; de los cuales 5 se encuentran en la provincia de Jaén. Las razones de esta concentración son varias; entre ellas, podemos destacar el auge económico de la provincia desde finales del siglo XIX por la mineria y el desarrollo industrial, así como por el hecho de tratarse de una de las provincias que posteriormente sufrieron con mayor intensidad la destrucción de la guerra civil, y que se encontraron, como consecuencia, dentro de las actuaciones prioritarias de Regiones Devastadas durante la posguerra.

La razón de ser del itinerario se encuentra en la propia lógica geográfica del abasto de poblaciones, que obligó a su implantación en ciudades medias para compensar las deficiencias de un sistema infraestructural de transportes que dificultaba la movilidad de los productos perecederos y aconsejaba su venta inmediata a la población. Esta lógica geográfica también se aplicaba a su localización en las ciudades, como era el caso del mercado de abastos de Úbeda que abre nuestro recorrido. Generalmente, estas experiencias de la modernidad buscaron su sitio extramuros, aprovechándose de una mejor accesibilidad para los sistemas de distribución, emplazándose como auténticos caballos de Troya de la modernidad a la espera del asalto a la ciudad histórica. 


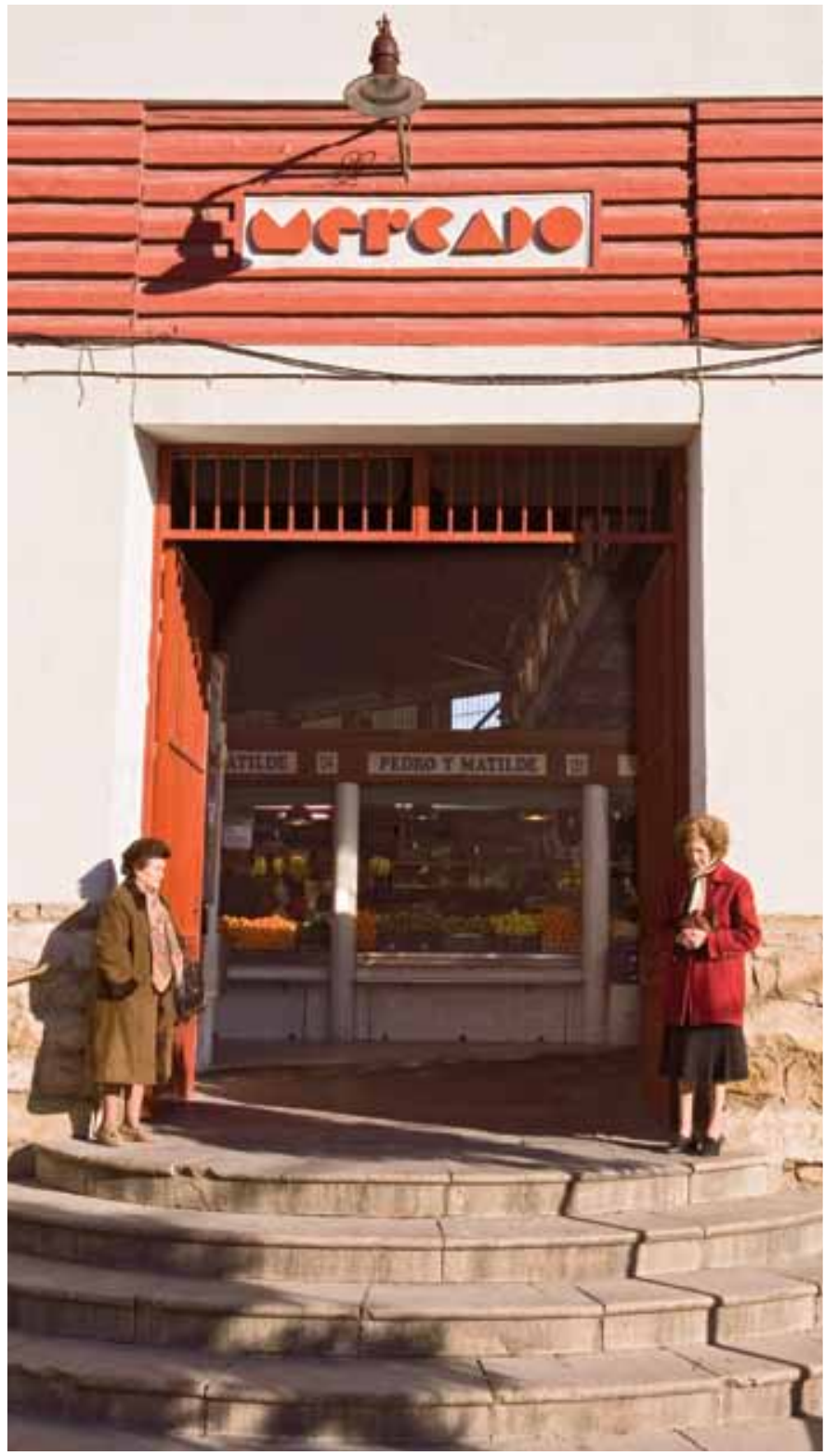

Vista del acceso principal al mercado de abastos de Úbeda Fuente: Fondo Gráfico IAPH

El mercado se consolidó como espacio público por excelencia en pueblos y ciudades, generador de una poderosa identidad contemporánea

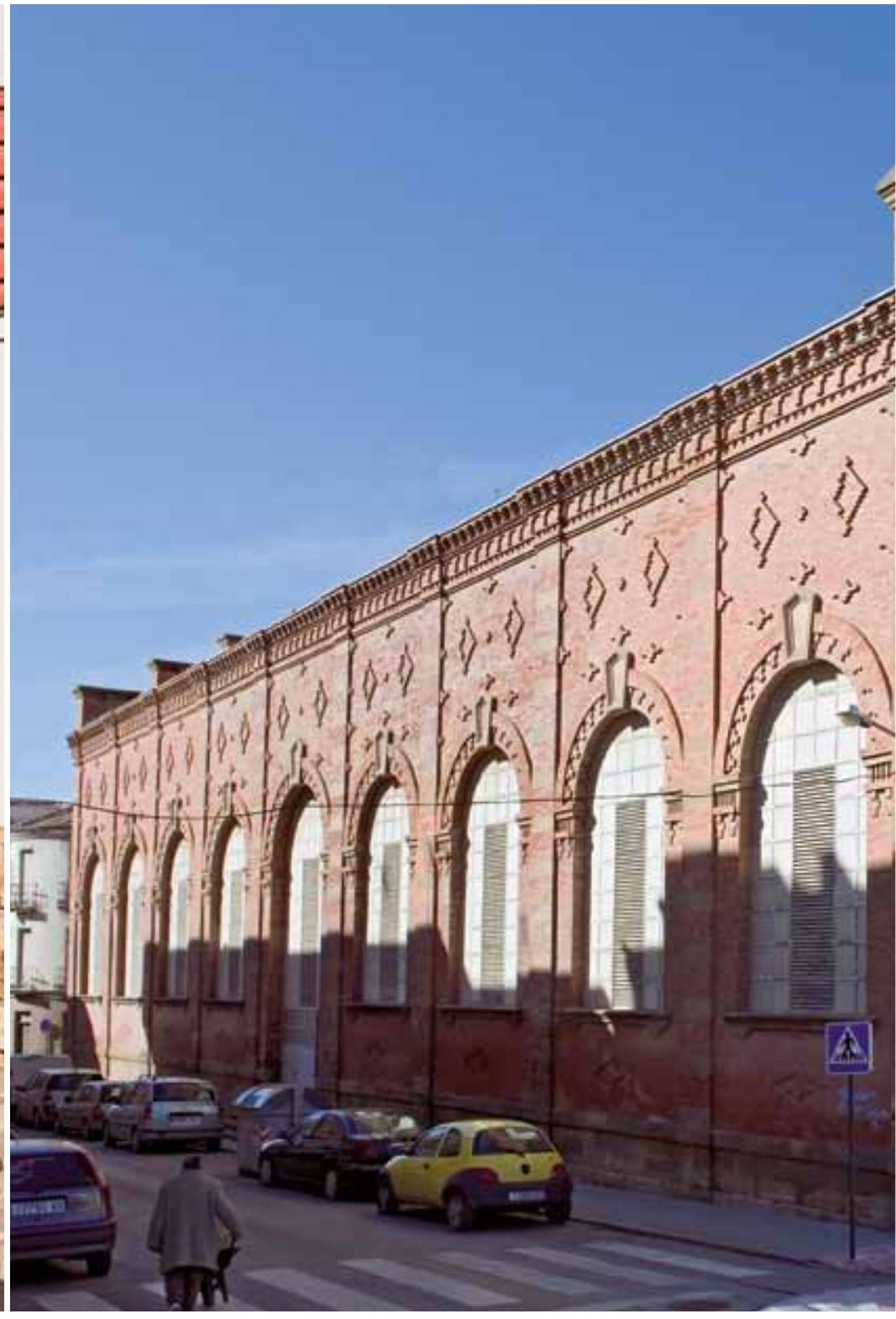

Vista exterior del mercado de abastos de Linares

Construido entre 1933 y 1936 por el arquitecto Luis Casanova Vila, el mercado de abastos de Úbeda se presenta como respuesta a los condicionantes topográficos del lugar. Un desnivel acusado entre el acceso principal de la plaza Gallego Díaz, enfrentado a la calle de Montiel como salida principal del centro histórico y la calle posterior de San Nicolás, obliga al edificio a dotarse de un potente zócalo de piedra, sobre el cual se alza el volumen enfoscado en blanco de la planta principal del mercado.

Sobre esta superficie se dibujan, a modo de manifiesto, rasgos netamente característicos de la arquitectura racionalista republicana, y que lo emparentan epidérmicamente con el ya mencionado mercado de Algeciras: la agrupación de los huecos en fachada, formando bandas horizontales remarcadas con la labra del ladrillo y con el color rojo intenso, son el elemento más caracteristico de la composición. De esta manera expresiva, se mostraban los paradigmas estéticos del movimiento moderno, 

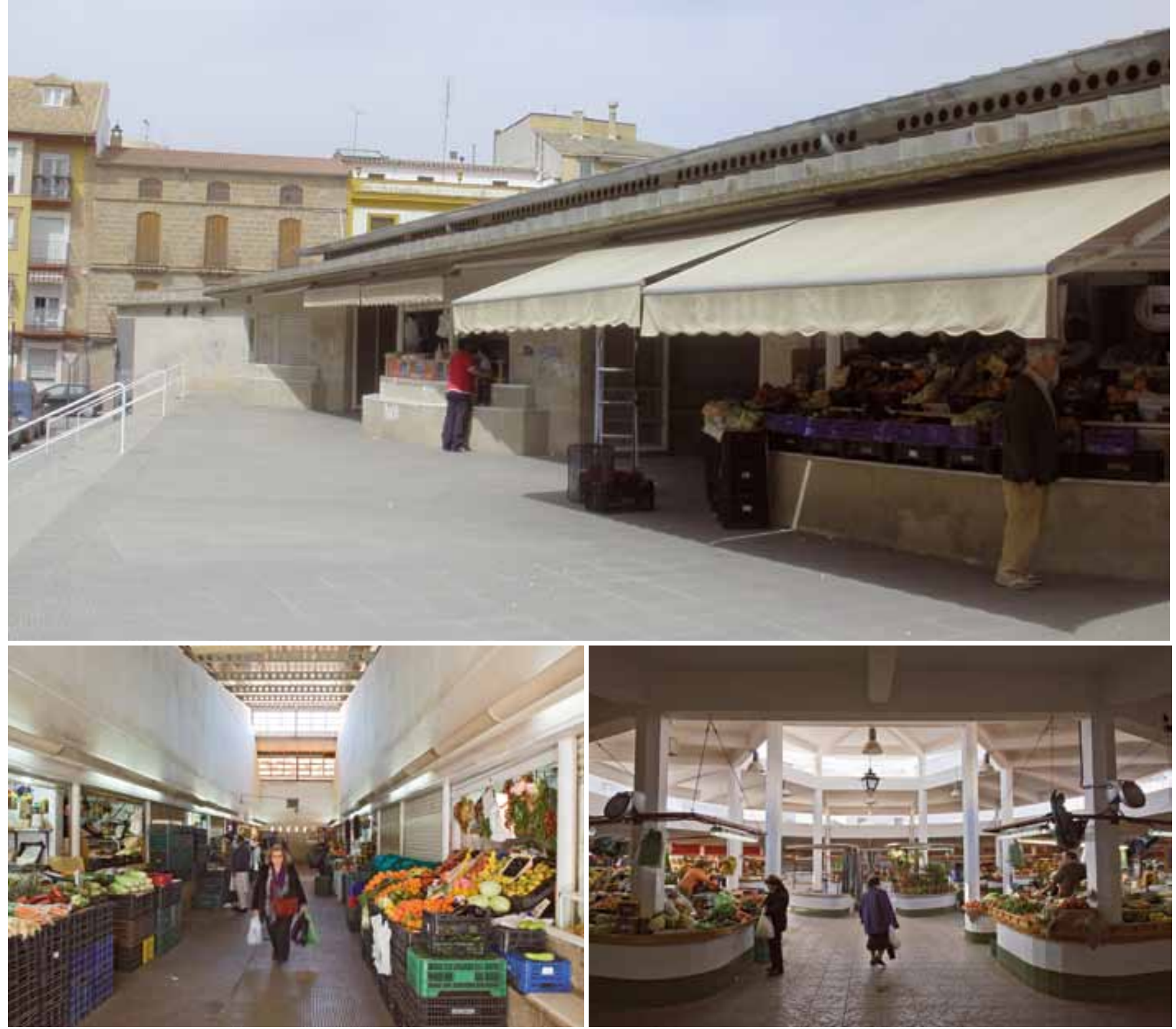

Vista exterior del mercado de frutas y verduras de Linares Vista interior del mercado de frutas y verduras de Linares Fuente: Fondo Gráfico IAPH

Espacio central del mercado de abastos de Andújar

hasta 1906, fecha de terminación del edificio, esta sencilla pieza es una muestra del pragmatismo ingenieril y arquitectónico con que se resolvía la cuestión del abastecimiento de poblaciones y uno de los escasos ejemplos de mercados promovidos en Andalucía durante el periodo de la Restauración. Un espacio de una sola nave de gran altura, cubierto con livianas cerchas metálicas, en cuyo interior se organiza la actividad comercial siguiendo un modelo absolutamente racional e ideal, con dos corredores centrales de distribución, más una galería perimetral, para facilitar el acceso de clientes y mercancias a los puestos de vendedores.

Hacia el exterior, el trabajo del arquitecto municipal Francisco de Paula Casado y Gómez deja entrever las conexiones del edificio con la arquitectura industrial de la época, evidenciadas en primer lugar por la elección del material. Un muro de mampostería revestido de ladrillo a cara vista constituye el cerramiento del edificio, en un gesto de modernidad adecuado en una inspiración nostálgica a la arquitectura ecléctica, presente en los motivos geométricos de los juegos de la labra, asi como la inclusión puntual de elementos ornamentales de piedra en los frontones del edificio y en la clave de los arcos de medio punto que enmarcan los huecos.

Justo en la acera opuesta de esta exquisita muestra de la arquitectura de inicios de siglo XX, encontramos el nuevo mercado de frutas y verduras construido en 1986 por el arquitecto Francisco Torres, pensado inicialmente para suplir las carencias del mercado de abastos original. La construcción de este edificio ejemplificó el final de la obsolescencia funcional que aconteció en los años 60 y 70 del pasado siglo, trasladando el comercio de las grandes ciudades y ciudades medias desde el ámbito tradicional de los mercados de abastos a los supermercados e hipermercados. Un proceso que vino asociado al auge de los productos de conservas y congelados, que entraron en los hogares andaluces modernizando modos de vida y modificando pautas de consumo.

Este nuevo edificio del mercado de frutas y verduras de Linares aparece claramente enmarcado, por tanto, dentro de una nueva sensibilidad acerca de la ciudad histórica, promoviendo el comercio 


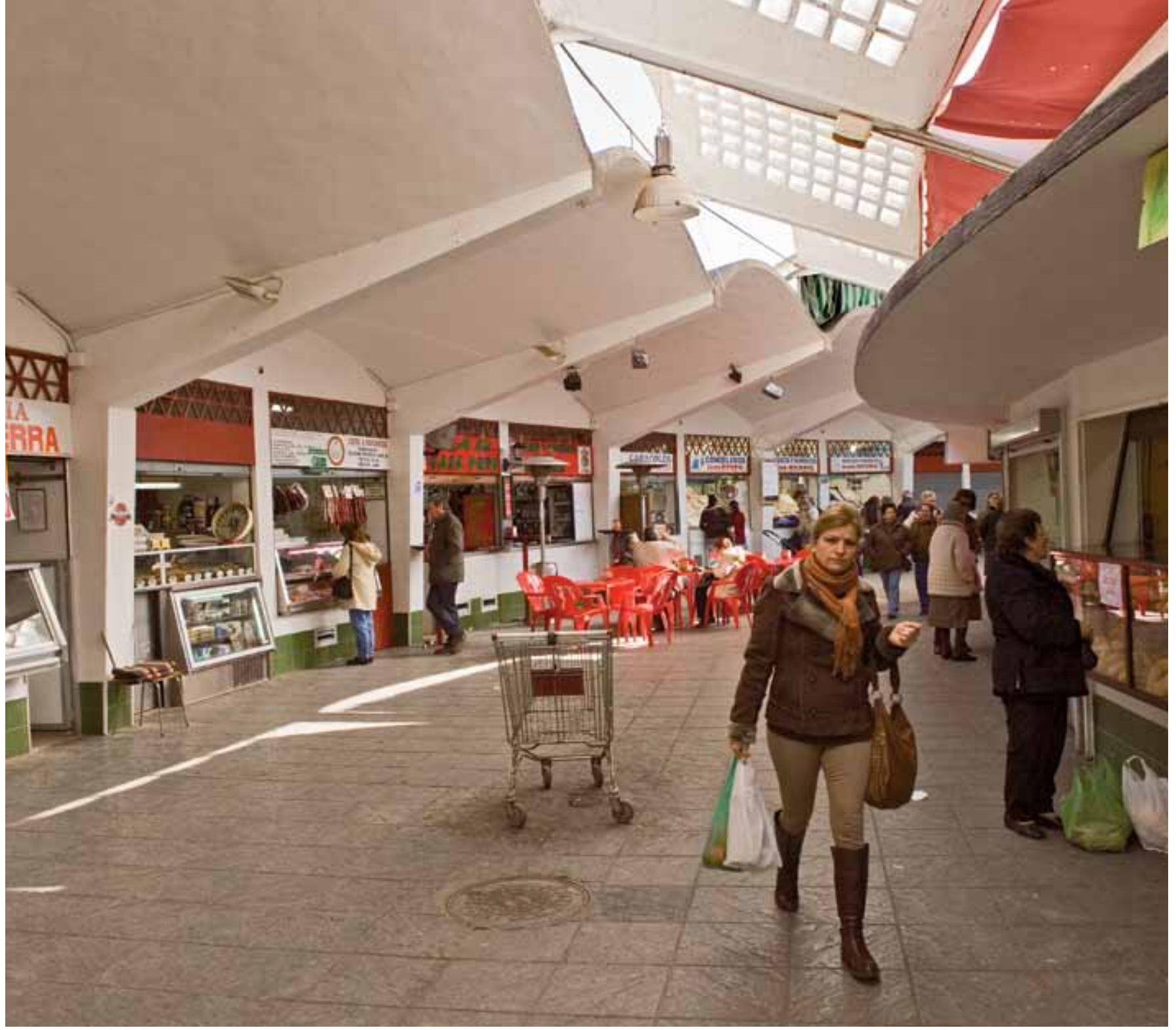

Calle interior del mercado de abastos de Andújar.

de proximidad y la recuperación de la significación histórica del mercado como espacio público. El planteamiento del edificio resulta especialmente comprometido con su dimensión urbana, invirtiendo el concepto de mercado cerrado para convertirse en espacio público registrable, a modo de calle cubierta, que se retranquea respecto de la alineación a la calle Santiago y ofrece el escalonamiento del espacio público de su fachada principal como gesto para consolidar la vocación cívica del espacio que lo separa del antiguo mercado de abastos.

Esa disposición urbana se acompañaba asimismo de un planteamiento tipológico innovador, en el que se procuran circulaciones independientes para mercancías y público, que hacen del edificio un ejemplo notorio dentro de los mercados de última generación. Pero al referirnos a las vicisitudes de este tipo de espacios comerciales habremos de hacer una reflexión preocupada sobre el destino de este edificio, ya que incluso la arquitectura más reciente resulta presa de los vaivenes de tendencias que experimenta el sector. La irrupción de nuevos mayoristas en el sector alimentario ha supuesto una nueva crisis en el modelo de mercado municipal, que incluso ha abierto un debate ciudadano sobre su posible demolición. Algo que no deja de resultar sorprendente para un edificio con escasos veinticinco años de antigüedad.

La visita a la cercana ciudad de Andújar es muestra de la vitalidad de la que goza este tipo de edificios en otros contextos, una situación en la que a buen seguro contribuye la existencia de una idea de proyecto contundente, tal es el caso del mercado de abastos de la localidad. El caso de Andújar muestra cómo la investigación tipológica de inicios de siglo llevó a la reflexión seria sobre el modelo de planta centralizada, explorando en la dirección que habian marcado Manuel Sánchez Arcas y Eduardo Torroja con el mercado de abastos de Algeciras, anteriormente mencionado. En Andújar, el proyecto que el arquitecto Pedro Rivas Ruiz redactó en 1939 supo hacer uso de las posibilidades del hormigón armado para configurar una pieza de un marcado carácter racionalista, que abrió la puerta a la consolidación de una arquitectura moderna de 


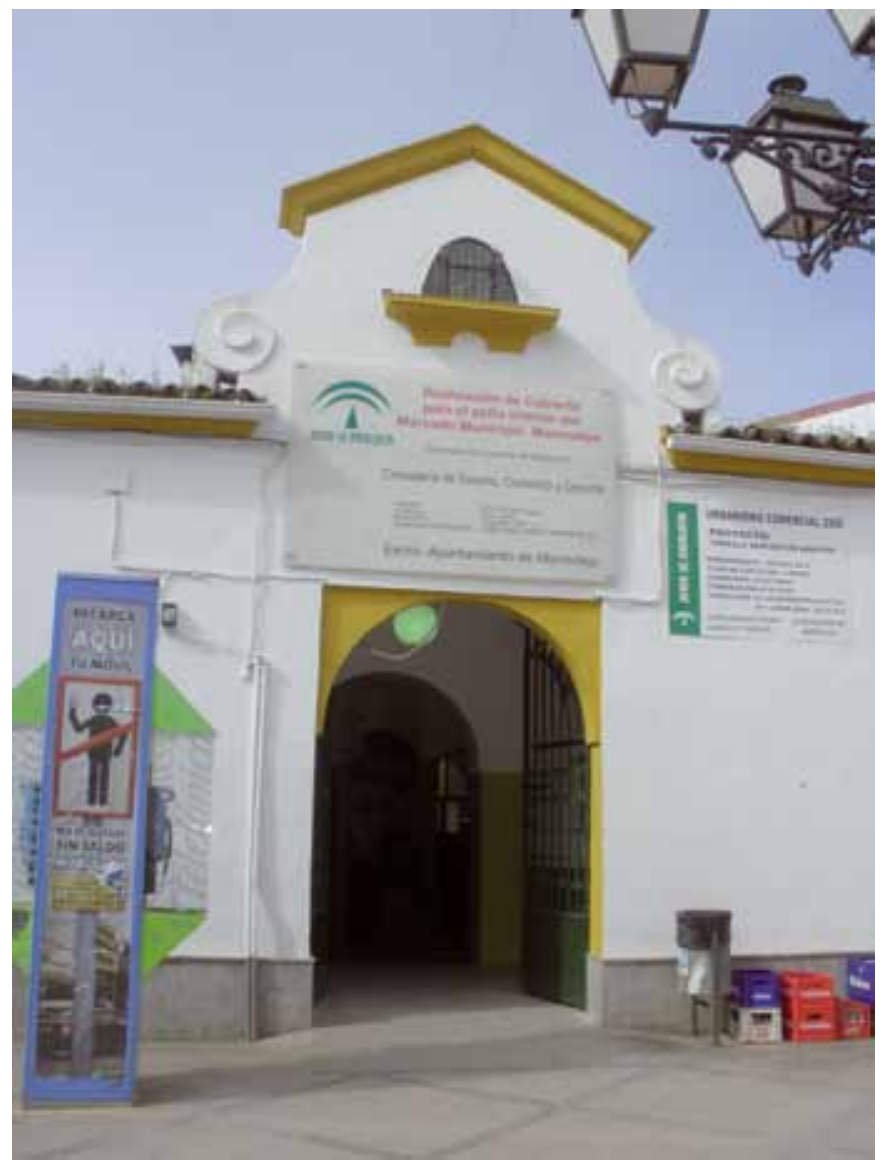

Fachada principal del mercado de abastos San José en Marmolejo. Fuente: Fondo Gráfico IAPH

notable factura en la ciudad, tales son los vecinos edificios de viviendas que ofrecen su fachada hacia la plaza de Rivas Sabater.

El espacio interior del mercado de Andújar es ejemplar en su organización comercial, que tiene una inmediata traducción en su funcionamiento como espacio público. Tras el paso de un estudiado umbral desde la calle, el edificio ofrece generosamente una primera calle curva, amplia y de traza circular, que alberga los puestos de carne y pescado, marcada por la presencia de un osado voladizo en hormigón armado que parte del anillo exterior y que cubria parcialmente el espacio abierto. Un acierto en el control de luces y sombras, que creaba una sensación de ambigüedad notoria hoy lamentablemente desaparecida por la adición de un voladizo adicional, que distorsiona la percepción del edificio. Tras ese anillo perimetral, un nuevo umbral marca el paso al corazón del edificio; un espacio cubierto por una estructura adintelada de hormigón armado, que en su centro se eleva en tres niveles ascendentes, al modo de tarta de pisos, permitiendo la iluminación y ventilación natural a los puestos de verduras y flores. Un espacio rotundo y desnudo, al que acompaña la vitalidad del uso cotidiano que de él hacen los ciudadanos.

La reflexión sobre la vida de los mercados en su condición de espacios públicos nos conduce a la última etapa del itinerario, en la localidad de Marmolejo, en el que es posible apreciar la trascendencia urbana de las decisiones de proyecto que trascienden lo meramente organizativo. El mercado, en esta ocasión, se pone al servicio de una empresa de construcción de identidad como fue la actuación del Servicio Nacional de Regiones Devastadas y Reparaciones promovido por el general Franco, que era paralela a la tarea de paliar el desabastecimiento de la población.

El mercado de abastos de San José en Marmolejo, obra del arquitecto Ramón Pajares Pardo en 1953, evidencia la complejidad programática y social de este espacio comercial, respondiendo en primer lugar a la provisión del equipamiento requerida mediante una estructura sencilla de puestos distribuidos alrededor de una modesta lonja cuadrada. Pero al mismo tiempo, el mercado se convertía en herramienta ideológica, dando un paso adelante en la consolidación de la imagen nostálgica que la dictadura pretendia imponer al territorio español en general y a las conocidas como Regiones Devastadas en particular.

Autarquía económica y control social se daban la mano, aprovechando la construcción del edificio para la creación de un espacio público abierto hacia la céntrica calle de Armando Palacio Valdés, hacia la cual el conjunto ofrece una fachada locuazmente autárquica. Como muestra de la raigambre antiurbana del pensamiento fascista, la secuencia urbana ideológica que arrancaba en la plaza del mercado y continuaba por la barriada de Regiones Devastadas desembocaba finalmente en el parque de la Libertad, que marcaba la entrada a la ciudad, y que resulta una expresión notoria de la monumentalidad que ansiaba el Régimen para la arquitectura pública.

Los casos de los mercados jiennenses ofrecen, por tanto, desde la modernidad sin ambages de Úbeda, hasta el ensimismamiento nostálgico de Marmolejo, la posibilidad de estudiar un proceso marcado por la confluencia de lucha ideológica, reflexión tipológica e identidad cívica. A través de este recorrido se descubre un relato privilegiado de la evolución histórica de esta tipología arquitectónica, que acompañó a la modernización territorial y social de nuestro entorno a lo largo del pasado siglo XX. Un proceso que, como hemos señalado, se muestra aún vivo a través de estos ejemplos, y del cual seguiremos reivindicando su valor para la potenciación de una identidad andaluza contemporánea.

\section{Bibliografía}

CASUSO QUESADA, R.; QUESADA GARCíA, S. (2006) La Estación de Autobuses de Jaén. Una topografía de su arquitectura. Sevilla: Consejeria de Cultura de la Junta de Andalucía, 2006

LANDROVE BOSSUT, S. (ed.) (2010) Equipamientos I: lugares públicos y nuevos programas. Registro DOCOMOMO ibérico, 1925-1965. Barcelona: Fundación Caja de Arquitectos, 2010

RUBIO DÍAZ, A. (2000) Mercados municipales de Andalucía. Sevilla: Dirección General de Comercio, Consumo y Cooperación Económica de la Junta de Andalucia, 2000 